\title{
Profumo di Vienna : 1809, le millésime autrichien de Stendhal
}

Jean-Jacques Labia

\section{OpenEdition}

1 Journals

Édition électronique

URL : http://journals.openedition.org/recherchestravaux/481

DOI : 10.4000/recherchestravaux.481

ISSN : 1969-6434

Éditeur

UGA Éditions/Université Grenoble Alpes

\section{Édition imprimée}

Date de publication : 15 décembre 2011

Pagination : 127-146

ISBN : 978-2-84310-215-8

ISSN : 0151-1874

\section{Référence électronique}

Jean-Jacques Labia, "Profumo di Vienna : 1809, le millésime autrichien de Stendhal », Recherches \& Travaux [En ligne], 79 | 2011, mis en ligne le 15 juin 2013, consulté le 08 septembre 2020. URL : http:// journals.openedition.org/recherchestravaux/481; DOI : https://doi.org/10.4000/recherchestravaux. 481 
Jean-Jacques LABIA

Université Paris X - Nanterre

\section{Profumo di Vienna : 1809, le millésime autrichien de Stendhal}

\section{La campagne de I809 et l'occupation française à Vienne : Stendhal et Cadet de Gassicourt}

Pour Beyle, tout commence ici par une vue des Alpes, quand les routes de l'Est s'infléchissent vers le Sud, pour une allègre métonymie : «Ces Alpes étaient pour moi l'Italie.» Il n'était pourtant que sur la route d'Augsbourg, par une «journée charmante». La lettre de Donauwerth à Félix Faure, datée du I6 avril I809, décrit l'épiphanie au présent : "J'aperçois tout à coup les Alpes : moment de bonheur. " Journal et correspondance échangent leurs fonctions. Les lettres du voyageur hébergent dans l'urgence un journal dont le destinataire doit devenir le gardien vigilant : "J'ai eu l'idée d'écrire mon journal le plus possible, et de t'en envoyer les feuilles à mesure; deux avantages, abréviation des lettres et sûreté. Seulement, ne perds pas les feuilles ${ }^{\mathrm{I}}$.»

Ainsi s'énoncent les thèmes que l'expérience et les campagnes, campagnes d'écriture aussi, ne cesseront de développer. Nous en retiendrons deux. D'abord une curiosité ouverte, qui dévalue ce que le voyageur en mission laisse derrière lui, dans son dos, sans regret comme il le constate explicitement : «ma passion pour Paris est bien diminuée». Et bien sûr l'approche d'une Italie toujours désirée dans une orientation nord-sud, mais avec cette singularité d'un seuil situé plus haut que prévu sur la carte!

I. Stendhal, Correspondance, t. I, éd. V. Del Litto, Gallimard, coll. «Bibliothèque de la Pléiade», 1962, p. 519 . 
Ce qu'il ignore sur le moment, c'est qu'un autre voyageur en mission lui aussi pour l'empereur tient, sur le même itinéraire et au même moment à quelques jours près, ce qu'il appellera aussi quand il l'imprimera en 1818 un «journal»:

Lépouvante qui précède une armée, la dévastation qui la suit, ne me laissant pas les moyens d'étudier les mœurs de nos ennemis, les productions naturelles et industrielles de leur pays. Que pouvais-je faire? recueillir des renseignements imparfaits, peindre des scènes isolées, conserver des aperçus fugitifs, jeter sur le papier des notes incohérentes; mais ces notes, ces aperçus, ces scènes fourniront peut-être un jour des matériaux à l'histoire. Ce journal, tout incomplet, tout décousu qu'il sera, ne renfermât-il qu'une page intéressante ou utile, je me saurai gré de l'avoir écrite. Dans un style négligé, dans un désordre apparent; je serai plus rapide, et je paraîtrai du moins original. Des faits et des sentiments, voilà ce que je m'efforcerai de reproduire pour mon agrément personnel; puissent mes lecteurs y trouver de l'instruction ou des plaisirs².

Ce «voyage», dirait Stendhal, est écrit par Cadet de Gassicourt en I8ı2, tel quel, dit-il. Seuls les "événements qui ont eu lieu depuis» en auraient retardé la publication. La question du "je» et de l'égotisme ne se pose guère, il n'est pas écrivain. Soulignons cependant sa formule : «je serai plus rapide», qui le rapproche de Stendhal par l'allure, ce "pas napoléonien" que saluera Nietzsche. Cadet veut saisir sur le vif, tel Stendhal dans une lettre à Pauline, «le drôle de désordre que la guerre produit ${ }^{3}$ ». Non sans se présenter, lui, en grand uniforme, revêtu de ses titres, décorations et crachats :

Pharmacien, Docteur de la Faculté des sciences, Membre de la Légion d'honneur, associé libre des Académies de Madrid, de Florence, de Turin, de la Société philotechnique de Paris, etc. etc. etc. ${ }^{4}$

Seul le fameux «etc.» serait ici stendhalien s'il n'était emphatiquement triple! On comprend mieux ainsi le retour tardif chez Flaubert du Premier pharmacien de l'Empereur en majesté grotesque, en autorité invoquée, en Aristote des potards. C'est à l'agonie d'Emma Bovary. Homais étale une érudition importune à propos d'une intoxication foudroyante "par des boudins qui avaient subi une trop véhémente fumigation». Il conclut : «Du moins c'était dans un fort beau rapport, composé par une de nos sommités pharmaceutiques, un de nos maîtres, l'illustre Cadet de Gassicourt 5 !»

2. Ch.-L. Cadet de Gassicourt, Voyage en Autriche, en Moravie et en Bavière, fait à la suite de l'armée française pendant la campagne de I809, L’Huillier, n I6, I8I8, p. VII-VIII.

3. Stendhal, lettre à Pauline Périer-Lagrange du 29 avril I809, Correspondance, ouvr. cité, p. 522 .

4. Ibid., p. vII.

5. G. Flaubert, Madame Bovary, Gallimard, coll «Folio», I972, p. 4I4. Homais veut briller aux yeux de l'immense Larivière, disciple de Bichat. Merci à D. Ferrer pour l'impromptu flaubertien! 
L'effet d'aubaine est évident pour Stendhal (on peut le nommer ainsi, il vient d'inventer cette signature pour Rome, Naples et Florence en I8I7) lorsqu'il découvre le livre de Cadet à sa parution en I8I8 sous le titre : Voyage en Autriche, en Moravie et en Bavière, fait à la suite de l'armée française. Il s'agit précisément ici de ce que Beyle a vu ou aurait pu voir lui-même en I809. C'est la meilleure occasion de recouper ses propres notes et impressions, en regard "des faits et des sentiments» rapportés par Cadet. Lauteur (I769-I82I) est de ses aînés, un homme du XviII ${ }^{\text {e }}$ siècle et des Lumières. Son portrait par Prud'hon au musée Carnavalet le montre en gandin un peu fat. Une source connue de Stendhal et rapportée par Martineau le dit né des amours de Louis XV avec la "belle» (sûrement) Madame Cadet, épouse d'un pharmacien membre déjà de l'Académie des sciences ${ }^{6}$. Cadet fils s'intéresse à la fabrication de la choucroute, à la bonne tenue des vaches les étables. Mais ce que retient Stendhal, il le note sur son propre exemplaire de Rome, Naples et Florence en I8I7, c'est "L'amour allemand à prendre dans Gassicourt"." L'auteur est du reste un savant voltairien, maçon, acide, narquois, curieux d'observations physiques et morales, que navrent les monuments religieux observés en traversant l'Est de la France : «D'Épernay à Strasbourg je n'ai pu voir, sans affliction, la multiplicité des croix récemment plantées par la superstition.» Les chronologies et les itinéraires des deux voyageurs se superposent. Beyle a quelques jours d'avance à Strasbourg, mais les dates coïncident à partir de Landshut, à Wels, Ebersberg, Melk, Sankt-Pölten, jusqu'à l'entrée dans Vienne le I3 mai. D'où la note au chapitre 54 de la Vie de Napoléon: "Voir le voyage à Vienne en I809 par M. Cadet-Gassicourt. Ce n'est pas une plume vendue ${ }^{8}$." Cadet revendique sa qualité de témoin oculaire, il garantit par exemple la véracité de la scène montrée par Gros dans ses Pestiférés de Jaffa: il y était. On voit que le livre intéresse doublement Stendhal, autant pour l'histoire en marche que pour les petits arrangements amoureux observés sur le terrain. Le jugement de valeur précité ne vient pas au hasard ni au jugé. Le contexte de la campagne de I809, note Stendhal, est bien celui d'une dégradation constatée du comportement de cette Grande Armée qui va bientôt se montrer «si égoïste et si corrompue à la campagne de Russie`». Cadet décrit ce que Beyle a pu voir : pillages, exactions, atrocités.

6. Stendhal, De l'Amour, éd. H. Martineau, Le Divan, I957, p. 439.

7. Ibid.

8. Stendhal, Vie de Napoléon, t. I, éd. L. Royer revue par V. Del Litto, Genève, Cercle du Bibliophile, I970, p. 228. Voir également pour le «tableau vigoureux et exact» recommandé par Stendhal au lecteur anglais, Chroniques pour l'Angleterre, t. I, éd. K. G. McWatters et R. Dénier, Grenoble, Publications de l'Université des Langues et Lettres de Grenoble, I980, p. 255 et suiv.

9. Ibid., p. 227. 
Une nuit, le pharmacien et ses compagnons, logés chez un chapelier, sont réveillés par des cris à l'étage. Cinq grenadiers ivres se déchaînent contre la famille de l'hôte :

L'un avait renversé sur les débris d'une commode une jeune femme grosse qu'il violait, l'autre faisait la même injure à la mère âgée de plus de soixante ans tandis que les autres contenaient et battaient les deux maris après les avoir dépouillés ${ }^{10}$.

Les menaces n'y feront rien. Aucune autorité militaire n'est à portée d'intervention. Les témoins doivent se résoudre à une retraite prudente «en gémissant de voir quel genre de haine éternelle de pareilles atrocités laissent partout où nous portons nos armes». La scène est à Wels, là même où les roues de la voiture de Beyle font "jaillir les entrailles des corps des pauvres petits chasseurs à moitié brûlés ${ }^{\mathrm{II}}$ ». L'auteur décrit aussi en ethnographe des petits faits vrais, anecdotes, observations directes, comme Stendhal les aime. Ainsi, toujours dans le registre noir, l'enterrement d'un indigent, à Wels encore : «il était clissé comme une bouteille de voyage. Chaque membre était entouré d'un rouleau de paille et serré comme une carotte de tabac». La Garde impériale, qui devrait être exemplaire, participe au pillage, tandis que les troupes alliées de la Confédération restent en bon ordre. Le groupe a un billet de logement chez un quincaillier, et Cadet se dit «assez heureux pour préserver sa maison de la rapine». Mais les hôtes pleurent au moment du départ, craignant «avec raison que les traînards ne respectassent point l'affiche de sauvegarde que j'avais mise à leur porte». C'est ce que Stendhal de son côté appelle à l'époque "le bacchanal ${ }^{12}$ ». Cadet voit "des hommes revêtus de la livrée de l'Empereur occupés à rouler des plats et des assiettes d'argent» dans un couvent de religieuses, pour conclure :

Qu'un soldat pille, les dangers auxquels il s'expose peuvent pallier sa faute; mais que les valets du souverain qui ne courent aucun risque prennent leur part du butin, c'est faire de leur maître un chef de brigands ${ }^{13}$.

Beyle et Cadet découvrent "l'horreur horrible ${ }^{14}$ » sur le champ de bataille d'Ebersberg, qui inspirera le Waterloo de la Chartreuse, en prenant la traverse :

J'ai vu pour la première fois les restes d'un champ de bataille, des morts, des blessés tout le long de la route, des débris de toute espèce, des champs dévastés, des chaumières découvertes et des fermes pillées. Ce spectacle nouveau pour moi affaiblit bien dans mon opinion le prestige de la gloire militaire.

Io. Ch.-L. Cadet de Gassicourt, Voyage en Autriche..., ouvr. cité, p. 67.

II. Stendhal, lettre à Félix Faure du 3 mai I809, Correspondance, ouvr. cité, p. 528.

I2. Stendhal, lettre à Pauline Périer-Lagrange du 29 avril I809, ibid., p. 523.

13. Ibid. p. 69.

I4. Stendhal, Journal, dans Euvres intimes, t. I, éd. V. Del Litto, Gallimard, coll. «Bibliothèque de la Pléiade», I98I, p. 534. 
Mon collègue, M. Vareliaud, me dit : quelle singularité! C'est un honneur de massacrer cent hommes dans un combat, et souvent c'est un déshonneur de donner la vie à un seul. Matière de bréviaire, lui répondis-je, comme notre maître Rabelais ${ }^{15}$.

Stendhal sépare sûrement dans sa lecture le bon grain pour lui (la saveur des anecdotes et des détails éventuellement macabres et horribles) de l'ivraie (la rhétorique morale) même s'il n’y a pas sans doute de réel désaccord sur le fond. Cadet, avec sa froideur de carabin, décrit des séquences cruelles : un cheval est frappé de plein fouet par un obus qui n'explose pas, le cavalier s'empresse de retirer la selle, l'obus explose! Un soir, il se couche, épuisé, près d'un feu à demi éteint, trouvant là «un amas de paille sèche sur laquelle paraît dormir un seul homme». Il tombe de sommeil. Au réveil, son camarade de bivouac n'a pas changé de posture :

Je reconnus l'uniforme autrichien, je regarde de plus près, il n'avait pas de tête : un boulet la lui avait enlevée la veille, et je ne m’en étais pas aperçu en me couchant près de lui ${ }^{16}$.

Ce détail servira plus tard à mettre en doute la posture et la pose des anciens témoins, quand le Stendhal fermement construit de I826, écrivant son second Rome, Naples et Florence (I827), se défiera des hâbleurs, voire même d'un «Français, non militaire» sur la "paille» d'une couche de hasard:

Monsieur, j’ai remarqué qu'un Français, non militaire, s'il est allé à la guerre, ne manque pas de raconter comme quoi il lui est arrivé une nuit de dormir sur un mort qu'il n'avait pas aperçu dans la paille, au fond d'une grange $[\ldots]^{17}$.

On reconnaît l'anecdote de Cadet sous la généralisation. Elle est d'ailleurs montée et sertie dans un subtil exercice d'ironie attribué à un cardinal homme d'esprit (Lante) qui dialogue fictivement avec l'auteur, ou son masque. La mise en doute n'épargnera pas quelques années plus tard le narrateur du Brulard, qui s'accuse au fil de la plume d'avoir laissé lui-même entendre plus haut qu'il était présent à Wagram. Et nous savons qu'il transpirait à l'heure même sous sa couette avec une fièvre de cheval. Le "génie du soupçon" est passé par là̀r${ }^{18}$. Il reviendra dans le Waterloo de la Chartreuse.

Le «voyage» n'en garde pas moins ses séductions pittoresques, voire romanesques. Le 9 mai, Beyle et Cadet sont à Melk, chacun de son côté : «une des

I5. Ch.-L. Cadet de Gassicourt, Voyage en Autriche..., ouvr. cité, p. 53-54.

16. Ibid., p. 228-229.

17. Rome, Naples et Florence, dans Stendhal, Voyages en Italie, éd. V. Del Litto, Gallimard, coll. «Bibliothèque de la Pléiade», I973, p. 432. Source inédite, semble-t-il.

I8. "Le génie poétique est mort, mais le génie du soupçon est venu au monde.» (Stendhal, Souvenirs d'égotisme, dans Euvres intimes, t. II, éd. V. Del Litto, Gallimard, coll. «Bibliothèque de la Pléiade», I982, p. 430) 
plus belles positions du monde», écrit le futur Stendhal, écrivain voyageur, à l'ami Félix Faure. Il ajoute ce commentaire troubadour : «la physionomie du paysage est d'accord avec le château où fut enfermé Richard Cour de Lion, qui en fait un des principaux ornements ${ }^{19}$ ». La vue lui semble digne de celles de Lausanne et de Bergame. De son côté, Cadet monte sur la plus haute tour de l'abbaye surplombant le Danube :

On me fit remarquer dans le lointain un vieux château en partie ruiné. C'est là, me dit-on, que fut enfermé Richard Coeur de Lion. Cette vue m’a rappelé successivement le troubadour Blondel, Sedaine et Grétry, qui l'ont rendu si intéressant, et la trahison de Léopold ${ }^{20}$.

Il faut observer que si le texte stendhalien date indubitablement de I809, celui de Cadet flotte entre I809 pour la temporalité interne, I8I2 date supposée de mise au net, et I8I8 date avérée de la publication... La tradition locale revendique le château de Deiteneg, visible de Melk, contredisant l'autre localisation des prisons de Richard au château de Dürnstein dans la Wachau, aujourd'hui préférée. Scott, dans sa série de "Waverley Novels», ne publiera son Ivanhoe que l'année suivante, en I8I9, pour ne rien dire du film justement célèbre de Richard Thorpe ${ }^{21} .$. Blondel est le protagoniste du livret que Sedaine écrit pour l'opéra de Grétry Richard Coeur de Lion (1784). Dans sa Vie de Rossini (II, IO3), Stendhal mentionne encore l'exécution de la «romance de Blondel» comme pierre de touche du vrai musicien qui comprendra Rossini ${ }^{22}$.

L'index rerum du livre de Cadet en fait un manuel commode. Il mentionne pour Vienne : «Ses promenades", dont bien sûr le Prater déjà décrit par Beyle sous le nom de Bombet dans son premier livre, les Lettres écrites de Vienne, etc., comme annoncées par la Bibliographie de la France, sa «brochure» dit-il, au titre interminable. La rubrique de Cadet : "Manière dont les femmes y font l'amour ${ }^{23}$ " n'étonnera pas le lecteur de Stendhal : cette source est citée telle quelle dans De l'Amour en $1822^{24}$. Elle est assez connue pour qu'il soit inutile d'insister ici. "Allemand, manière agréable et prompte d'apprendre la langue allemande", écrit Cadet, c'est sur l'oreiller auprès d'une beauté du cru, comme le recommandera (pour l'anglais) l'ineffable Tabard à Antoine Doinel dans Baisers volés ${ }^{25} \ldots$ On verra plus loin les dommages collatéraux de ces échanges

19. Stendhal, lettre du Io mai I809 à Félix Faure, Correspondance, ouvr. cité, p. 528.

20. Ch.-L. Cadet de Gassicourt, Voyage en Autriche..., ouvr. cité, p. 8o.

2I. R. Thorpe, Ivanhoe, 1952, avec l'inoubliable Rebecca de Liz Taylor, Joan Fontaine (Rowena), George Sanders (Bois-Guilbert) et Robert Taylor (le héros).

22. Stendhal, Vie de Rossini, t. II, éd. H. Prunières, V. Del Litto, E. Abravanel, Genève, Cercle du Bibliophile, 1968, p. I03.

23. Voyage en Autriche..., ouvr. cité, p. 286 pour le texte, p. 420 pour l'index.

24. Stendhal, De l'Amour, ouvr. cité, chap. XLviII, p. I47 et suiv.

25. Fr. Truffaut, Baisers volés, I968, Lonsdale et Seyrig sous les yeux de Léaud. 
linguistiques. "Amputations» : moins fréquentes qu'en France, mais non pas interdites comme on le croyait. Plus directement stendhalien, à l'horizon de la Chartreuse, l'article "Vivandière - Manière de vivre d'une vivandière». Quant à la mention "Haydn : célèbre compositeur de musique. Hommage que lui rend l'armée française", elle vient ici quatre ans après les Vies de Haydn, Mozart et Métastase. Quant à "Larrey» c'est bien sûr le chirurgien en chef de la garde impériale, un collègue de Cadet, qui possédait d'ailleurs son exemplaire du livre, avec inscription manuscrite (autographe) de son nom. Il fut légué à la Bibliothèque nationale par une demoiselle Dodue et on le trouve au fonds Larrey. Mais curieusement, c'est l'exemplaire premier et banal de la Bibliothèque nationale qui intrigue, avec ses passages cochés par un crayon anonyme : a-t-il quelques modestes secrets à livrer ${ }^{26}$ ? Larrey et sa geste, comme lorsqu'il sauve d'une mort ignominieuse, après sa propre enquête de police scientifique élégante et précise digne d'un Dupin ou d'un Holmes, "24 soldats blessés injustement accusés de lâcheté» : on s'apprête à les fusiller pour l'exemple, car on prétend qu'ils se seraient mutilés volontairement; Larrey prouve qu'ils ont tous été victimes du même matériel défectueux et dangereux. À l'occasion d'une "Belle opération dont il est mal récompensé», Cadet cite un de ses propres traits d'esprit, ce fameux esprit français glosé et non moins pratiqué par Stendhal. Un général a l'épaule fracassée; magistralement amputé par Larrey, il lui offre un diamant minuscule, de ceux qu'on appelle à l'époque «étincelle»:

- Eh bien! lui dis-je, si le général brûle pour vous du feu de la reconnaissance, il n'en montre qu'une étincelle ${ }^{27}$.

Mentionnons pour mémoire l'envers crûment réel aussi du décor amoureux, la "Prostitution" dont le livre brosse un tableau sans fard sous l'occupation française, en contrepoint épidémique des bonnes feuilles sur la variante autrichienne de l'amour allemand que Stendhal cite peu après la mort de Cadet : «Les maladies syphilitiques étaient assez rares à Vienne avant l'arrivée des armées françaises. Elles ont centuplé en un mois.» Côté Stendhal : le 29 décembre i8r9 est le "Day of Genius», la "First idea» de De l'Amour. Mais, ce même jour, il note aussi une «blenora apparence», que va bientôt confirmer une lettre à Mareste : sa première chaude-pisse "depuis I809" le millésime viennois. Le propos du potard, que Stendhal vient de lire de près (dès I8I8), se trouve simplement vérifié. Beyle, en homme de terrain, a payé de sa personne en contribuant activement aux statistiques des maladies vénériennes.

26. Stendhal aurait-il eu en mains cet exemplaire? Ou un stendhalien voyou mal surveillé? Le passage sur la vivandière, ainsi qu'un épisode de cheval volé, tous deux assez "chartreux", sont cochés. Que faut-il en penser?

27. Ch.-L. Cadet de Gassicourt, Voyage en Autriche..., ouvr. cité, p. 90. 
Il en garde un souvenir cuisant, quoique vif et sans rancune, quand vient la rechute dix ans plus tard. Drôle de petite madeleine, malgré tout!

Deux points au moins évoquent dans le récit de Cadet d'autres échos stendhaliens. Un détail double, sur les attitudes d'abord, concernant «la blague»:

Le grand mérite des courtisans oisifs est de blaguer avec vraisemblance. Aussi nous fatigue-t-on tous les jours de récits plus absurdes les uns que les autres ${ }^{28}$.

Ce motif, récurrent chez Stendhal, recoupe celui du «contraste de bravoure et de fatuité» observé par le pharmacien chez un "général de mélodrame» dont il dresse le portrait : Dorsenne, beau comme un Adonis, fort comme un Achille. Mais aussi, plus importante pour l'axiologie stendhalienne, la figure de Joseph II, le thème du gouvernement éclairé, en lutte contre les pouvoirs de l'Église, avec une anecdote sur "Ce prince outragé par les capucins» qui «insultent à sa cendre». Stendhal, héritier lucide des Lumières et «houzard de la libertée ${ }^{29}$ ", n’en déplaise à plusieurs, y reviendra dans les deux Rome, Naples et Florence à dix ans d'intervalle, en I8I7 et en I827. Mais c'est une autre histoire.

\section{L'Autriche ou Vienne? Stendhal et les Viennois}

Comment saisir l'identité autrichienne? Les Autrichiens eux-mêmes ne sont pas les derniers à embrouiller la question. Ainsi Rudolf Baehr, dans son bel article sur "L'Année autrichienne de Stendhal », décide de "considérer comme autrichien » le pays de Salzbourg en observant qu'il fait partie de l'Autriche» la première fois en 1805 et de manière définitive à partir de $1816^{30}$. Mais cet empereur Joseph II (qui règne de 1765 à I790), dans l'inscription d'Ermenonville qui célèbre son pèlerinage rousseauiste et sa modestie, pour avoir le 24 mai I777 "préféré aux palais cette simple chaumière » pour y dîner, et ainsi «ferme conservé l'égalité première", cet empereur d'Autriche ami des Lumières y est aussi nommé, en style inversif, «des Germains fortunés et le Père et le maître». Les Allemands et les Autrichiens seraient donc des Germains. Cependant les Allemands ne sont sûrement pas des Autrichiens. Quoique certains soient les sujets de l'empereur Habsbourg, empereur autrichien et donc lui-même aussi Allemand par certains de ses sujets. Mais les Autrichiens pourraient n'être après tout que des Allemands du Sud? C'est d'ailleurs un vénérable sujet de

28. Ch.-L. Cadet de Gassicourt, ouvr. cité, p. 262.

29. Loin d'être «les plus bas des hommes", les gens de lettres sont "les houzards de la liberté; ils sont tous les jours au feu, il faut bien qu'ils reculent quelquefois» (Voir Rome, Naples et Florence en I8I7, Julliard Littérature, I964, note p. I26).

30. Voir R. Baehr, "L'année autrichienne de Stendhal», Stendhal Club, nº 96, 198I-1982, p. 356-373. 
plaisanteries et de motifs littéraires dans l'espace allemand que ces affinités et leurs contraires. Par exemple chez Thomas Mann, le second mariage de Tony Buddenbrook avec un philistin bavarois, certes pittoresque, ne peut être qu'une mésalliance, que les hanséatiques lübeckois regardent de haut, du haut de la carte, comme une déchéance... Cependant, à un moment précis de l'histoire, la petite et libre Autriche, née des traités de paix suivant la Grande Guerre (Saint-Germain, Trianon), pourra devenir, et ce sera finalement pour cinq précieuses années, entre la prise de pouvoir d'Hitler en 1933 et l'Anschluss de 1938, "une sorte d'arche de Noé de la culture allemande», selon la belle formule de Musilis ${ }^{3}$.

La Vienne de I809 rayonne de séduction. Dans une lettre à Pauline, Beyle déclare : "Vienne est un des plus beaux climats que j'aie vus ${ }^{32}$." Comme dirait Montaigne, d'une température de pays qui fait les hommes... amoureux, à en croire une lettre à Félix dès l'arrivée : «Le séjour de Vienne me charme et produit une singulière tristesse; trop de penchant à l'amour : une jolie femme à chaque pas ${ }^{33}$.» Les premiers pas originaux et personnels de Stendhal (Bombet) en écriture publiée dressent d'ailleurs un tableau de la ville dans la seconde partie du premier chapitre des Lettres écrites de Vienne sur Haydn [...] car tel est le début du titre de la brochure de I8I4. Lettres en partie volées, empruntées, réécrites, mais par quelqu'un qui sait de quoi il parle. En somme, "Vienne est une ville charmante ${ }^{34}$.» Le Prater est pour le voyageur sensible "la première promenade du monde" à hauteur, pour les "promenades" de ce que représente pour le paysage "la vue de la baie de Naples». Avec le Danube, le Leopoldsberg, le Kahlenberg - telles à Grenoble les montagnes au bout de la rue, ces hauteurs dont il regrette l'absence à Paris avant de découvrir les coteaux modérés de Saint-Cloud, d'Andilly et de Montmorency. Si l'esprit n'y règne pas comme à Paris, ni la raison comme à Londres, néanmoins :

une certaine réserve, qui fait partie de la politique savante de la maison d'Autriche, a porté les peuples vers des plaisirs plus physiques, et moins embarrassants pour ceux qui gouvernent ${ }^{35}$.

Bombet souligne d'emblée les liens de la maison régnante «avec l'Italie, dont elle possède une partie; plusieurs de ses princes y sont nés. Toute la

3I. "L'Écrivain dans notre temps", conférence du I6 décembre 1934 à Vienne pour les vingt ans du Schutzverband deutscher Schriftsteller, dans R. Musil, Essais, trad. Fr. Jaccottet d'après l'édition d'A. Frisé, Seuil, 1984, p. 284.

32. Lettre du Is juin I809, Correspondance, ouvr. cité, p. 37I

33. Stendhal, lettre à Félix Faure du i8 mai 1809, Correspondance, ouvr. cité, p. 532.

34. Stendhal, Vies de Haydn, Mozart et Métastase, Genève, Cercle du Bibliophile, I970, p. II.

35. Ibid., p. I3. 
noblesse de Lombardie se rend à Vienne pour solliciter de l'emploi, et la douce musique est devenue la passion dominante des Viennois».

Donc le sud italien est bien présent, en deçà des montagnes qui en dessinaient le seuil ou la promesse. Cet alliage, dû au génie du lieu, est ici le secret du charme féminin :

un teint superbe sert de parure à à des formes élégantes : l'air plein de naturel et quelquefois un peu languissant et un peu ennuyeux des Allemandes du Nord, est mélangé ici d'un peu de coquetterie et un peu d'adresse (effet de la présence d'une cour nombreuse). En un mot, à Vienne, comme dans l'ancienne Venise, la politique et les raisonnements à perte de vue sur les améliorations possibles étant défendus aux esprits, la douce volupté s'est emparée des cœurs ${ }^{36}$.

D'où la musique qui triomphe «ici même sur la hauteur allemande» avec Haydn, Mozart, et Cimarosa. Ce dernier est également mentionné par Cadet de Gassicourt : "On se rendit à la salle de spectacle, où l'on donnait Il Matrimonio secreto pour la cinquième fois ${ }^{37}$. On voit que le goût de Beyle est partagé à l'époque - mais sans doute plus durable : comme l'antique princesse de la Chartreuse, il aimera toujours «la musique surannée» que perpétuent les recueils d'Arie antiche. D'ailleurs Bombet insiste sur ce point : «En musique, comme pour beaucoup d'autres choses, hélas! je suis un homme d'un autre siècle ${ }^{38}$." Il clôt son portrait de Vienne en rappelant que "c'est à Prague que Cimarosa a composé son Matrimonio segreto». Il y va lui aussi revoir l'opéra du Napolitain, "mais je le sais par cœur" ajoute-t-il. Cadet raconte de son côté plus d'une anecdote sur Haydn, notamment sur la garde d'honneur qui protège en i80s le compositeur apeuré lors d'une première reddition de Vienne aux Français ${ }^{39}$.

On voit par le détail pragois que si le centre de l'Autriche est à Vienne, la circonférence est variable, et peut-être nulle part. Cadet-Gassicourt décrit le Prater comme un lieu de rencontre unique entre les milieux sociaux, mais aussi :

vingt peuples et vingt costumes différents : ce sont des Turcs, des Grecs, des Bohémiens, des Hongrois, des Cosaques, des juifs, les uns coiffés d'un turban, les autres d'un béret; les Israélites barbus; les anabaptistes en lévite brune, et la tête couverte d'un grand chapeau ${ }^{4}$.

Des orchestres (vingt ou trente, dit-il!) distribués dans la forêt accompagnent musicalement la promenade : le "Chasseur vert» de Stendhal n'est

36. Ibid., p. I4.

37. Ch.-L. Cadet de Gassicourt, Voyage en Autriche..., ouvr. cité, p. 213.

38. Vies de Haydn..., ouvr. cité, p. 399.

39. Ibid., p. 260.

40. Ch.-L. Cadet de Gassicourt, Voyage en Autriche..., ouvr. cité, p. I77. 
pas loin. Une gourme nationale peu stendhalienne empèse toutefois certains jugements. Ainsi quand sur le fond de ce «bizarre assemblage» se promènent "les élégans de la ville habillés à la française mais avec toujours dans leur mise et dans leur maintien quelque chose de tudesque».

Ce carré magique viennois, dont Stendhal retient surtout l'orientation nord-sud ${ }^{4 \mathrm{r}}$, et Cadet l'ouverture de l'occident à un orient bigarré, sera dénoncé par Musil comme un sophisme mystifiant. C'est le paradoxe de «l'explication patriotique» du concept de culture autrichienne :

Elle dit toujours ceci : nous sommes si doués, l'Orient et l'Occident fusionnent en nous, ainsi que le Nord et le Sud, une pluralité magique, un admirable croisement de races et de nations, toutes les cultures collaborant et s'intégrant féeriquement, voilà où nous en sommes ${ }^{42}$.

Pour les personnages autrichiens (d'ailleurs viennois) de L'Homme sans qualités, le Reich berlinois de I9I3 n'est qu'un parvenu : c'est le thème de «l'Action parallèle», autour du grand et pacifique jubilé de l'empereur FrançoisJoseph (soixante-dix ans de règne en I9I8; mais il n'y sera plus) opposé au petit jubilé belliqueux du Prussien tard venu, Guillaume II (trente courtes années). Mais finalement l'État modèle autrichien n'a pas «dépassé l'état de scandale européen, juste après la Turquie». Il faut se souvenir de la formule d'alors, attribuée au tsar Nicolas ${ }^{\text {er }}$, qui voyait ce pays comme "l'homme malade de l'Europe». Musil brosse un tableau sans complaisance du vide culturel autrichien à l'heure du dépôt de bilan, après la mort de FrançoisJoseph en 1916, la défaite et la fin de l'empire en I9I8, dans son article intitulé "L'Autrichien de Buridan». Seule solution pour une collectivité dépourvue de «talent», faute d'Autriche, on célébrera les vertus de l' "Autrichien":

Puisque l'Autriche n'en était pas douée, il y eut l'Autrichien doué et cultivé (dans une proportion qui nous assurera une place relativement bonne en Allemagne), mais il n'y eut pas de culture autrichienne. La culture d'un État consiste dans l'énergie avec laquelle il rassemble des livres et des tableaux et les rend accessibles, avec laquelle il installe écoles et lieux de recherche, offre aux hommes de talent une base matérielle et leur assure une impulsion grâce à la puissance de sa circulation sanguine ${ }^{43}$.

4I. Voir cependant : «je ne suis pas sûr de ne pas regretter ce que verront ceux qui iront en Bohême et en Hongrie, et peut-être en Turquie" (Lettre à Félix Faure du I8 mai I809, Correspondance, ouvr. cité, p. 53I). Et bientôt sa mission en... Hongrie (explorée sur des documents inédits par E. Williamson).

42. R. Musil, «L'Autrichien de Buridan», traduit par Fr. de Buzon, L'énergumène, revue dirigée par G.-J. Salvy, nº 5, 1975, p. 6. Et non pas «L'Autriche de Buridan » comme fâcheusement dans R. Musil, Essais, ouvr. cité, p. 85 et suiv.

43. Ibid., p. 7 . 
Zweig constate dans Le Monde d'hier, écrit au Brésil, qu'on en est arrivé là dans les années de la Seconde Guerre mondiale. La ville de Marc Aurèle, la métropole deux fois millénaire où il est né, où il a été élevé, il a dû la quitter comme un criminel «avant qu'elle ne fût humiliée jusqu'à n'être plus qu'une ville de province allemande», lui dont la langue maternelle était d'ailleurs en un sens l'italien, par sa mère née à Ancône, du côté des artichauts et du risotto plutôt que des Würze... Il s'était ainsi trouvé en sympathie profonde avec le pianiste et compositeur Ferrucio Busoni, un frère en déchirement. C'était en Suisse, après l'entrée en guerre retardée de l'Italie en I9I5, aux côtés de la France et de l'Angleterre, suivant le renversement d'alliances préparé secrètement qui vaudrait ensuite à l'Italie l'acquisition du port austro-hongrois de Trieste, où Busoni avait d'ailleurs été élevé... Busoni était en effet "Italien de naissance et d'éducation, Allemand par le choix de son genre de vie». Il refusait pour le moment de se produire comme virtuose dans tous les pays belligérants. Il fut ensuite titulaire de la chaire de composition à l'Académie des Arts de Berlin, où Schoenberg, le maittre de la seconde école de Vienne devait lui succéder, ayant "fui à temps à l'étranger" selon une logique toute musilienne de l'économie culturelle... Zweig rappelle les doutes de son ami :

Quelle est ma patrie? me demanda-t-il un jour. Quand je rêve la nuit et que je m'éveille, je sais que j’ai parlé en italien dans mon rêve. Et quand, ensuite, je me mets à écrire, je pense avec des paroles allemandes ${ }^{44}$.

Il le rencontre à une heure avancée de la nuit dans la grande salle du buffet de la gare de Genève, ayant vidé solitairement deux bouteilles, étrange image légendée par Busoni lui-même :

Il faut parfois s'étourdir, sinon on ne le supporterait pas. La musique ne le peut pas toujours, et le travail ne nous visite qu'aux bonnes heures ${ }^{45}$.

Malgré son scepticisme en face de l'"Autrichien de Buridan», hésitant entre l'Allemagne et l'Europe du Milieu, Musil doit bien avouer qu'un parfum de Vienne lui revient de très loin par le double truchement de Stendhal et de cet autre voyageur longuement cité dans De l'Amour. Il s'émerveille des citations attribuées par Stendhal à Cadet-Gassicourt, sur «l'amour allemand» observé dans le contexte autrichien, qui aurait "quelque chose de mystique». Il se demande même si Stendhal n'aurait pas «inventé» ces fragments surprenants ${ }^{46}$. Car si on le lit dans ce contexte musilien, le texte de Stendhal, inactuel, salué par Nietzsche, et enfin devenu canonique en terre

44. St. Zweig, Le Monde d'hier, souvenirs d'un Européen, Albin Michel, 1948, p. 323.

45. Ibid.

46. R. Musil, Gesammelte Werke in neun Bänden, éd. A. Frisé, t. 5, Rowohlt, I978, p. 1943. Il trouve trop beau ce passage qu'il cite en français, où Stendhal cite lui-même Gassicourt. Musil commente : «erfunden? Ich weiss es nicht» («inventé? Je l'ignore)». 
germanique, commente par avance la relation des jumeaux siamois Ulrich et Agathe dans L'Homme sans qualités. Et Musil de s'émerveiller : "Le livre que j'écris serait donc un peu allemand?» - allemand donc ici, et non pas autrichien. L'identité autrichienne reste une qualité refusée comme une réduction à un provincialisme culturel.

Entre désespoir et optimisme, Doderer reprend implicitement, mais indubitablement, la problématique musilienne, dans le nouvel après-guerre. Nous sommes cette fois en 1945, dans le Livre vert, tel du moins qu'il l'édite luimême testamentairement, pallidus morte futura, dans la publication anthume d'une partie de ses journaux sous le titre de Tangentes (1964), deux ans avant une mort annoncée. L'espace est pensé en termes d'échanges et d'intersection, Vienne devient une "ville invisible» au sens de Calvino, de l'espèce des «città sottili», villes "effilées" ou plutôt "déliées" dans leur calligraphie aérée où les "pleins» s'abolissent ${ }^{47}$. Son insularité l'inscrit dans la figure d'une antique cité de l'archipel grec :

À première vue, l'Autriche n'a que deux possibilités : elle peut opter pour l'Allemagne ou pour l'Europe. Ces deux choix n'ont rien d'autrichien. C'est pourquoi nous préférons redevenir une gare de transit pour tous, ou une "plaque tournante» selon l'expression de d'Ormesson dans le Figaro en 1938. C'est également le meilleur moyen de laisser aussi loin que possible derrière nous le concept quantitatif de nation. L'Autriche est par essence un État à l'antique, une Jólís, que Vienne contient tout entière, un point d'intersection, pas une étendue. Il en fut toujours ainsi, même quand elle s'annexait des couronnes et des pays. La part de matérialité grossière est infime dans l'essence nationale autrichienne ${ }^{48}$.

On se console comme on peut dans la petite Autriche effilochée. Après les soustractions territoriales, le spirituel est recommandé.

\section{Hallein, les saisons et le millésime autrichien : une topique nouvelle}

Le Calendrier de Stendhal, selon Martineau, montre combien le stendhalien a horreur du vide - comme Stendhal d'ailleurs, qui regrette d'avoir perdu «la suite et la fin» de ses «journaux» de 1809 après l'entrée du 20 novembre et jusqu'au Is février I8Io. Martineau pense que c'est en fin décembre I809 "que l'on pourrait placer à la rigueur une visite aux mines de sel de Hallein ${ }^{49}$ ". Nous savons désormais, grâce à l'enquête sur le terrain du Salzbourgeois

47. I. Calvino, Le Città invisibili, Turin, Einaudi, 1972.

48. H. von Doderer, Tangenten, Aus dem Tagebuch eines Schriftstellers, Grünes Buch [1964], Munich, Deutscher Taschenbuch Verlag, 1995, p. 364. Je traduis.

49. H. Martineau, Le Calendrier de Stendhal, Le Divan, I950, p. IO2. 
Rudolf Baehr ${ }^{\text {so }}$, que Stendhal y est bien allé, et même deux fois. C’est au début du mois suivant, sur le chemin du retour en France, les 5 et 6 janvier I8Io. "Peu nous importe», ajoutait Martineau (par dépit amoureux?), "que Stendhal soit réellement allé à Salzbourg et à quelle date». Coe, sans savoir encore, observait que la mine, située à flanc de montagne, devait être peu accessible en hiver, cet argument plaidant en faveur d'une visite fictive. Le vrai n'est pas toujours vraisemblable. Mais il avait le nez creux lorsqu'il s'étonnait, dans cette hypothèse, que le texte de 1825 (Le Rameau de Salzbourg) puisse mentionner la petite localité de Golling à I2 kilomètres au sud de Hallein, et tant de détails sur la visite des mines de sel sans disposer de notes... Tout cela quinze ans après!

En tout cas, les montagnards de I8Io sont bien là pour ce voyage d'hiver. Faut-il imaginer Stendhal en raquettes ou à peaux de phoque? Le livre en témoigne, semblable, dit Baehr, à un vieux missel. Il contient environ I4 ooo noms de visiteurs, et est maintenant conservé au Keltenmuseum (musée des Celtes) de Hallein. La mine, l'une des plus anciennes du monde, 7000 ans dit-on, située à Bad Dürrnberg, dans la "vallée du sel», à 835 mètres d'altitude, est désormais reliée par funiculaire à Hallein. Elle est la source de la prospérité de l'émirat des princes évêques et des splendeurs baroques de Salzbourg : c'est l'"or blanc». La serge grise, mentionnée par Stendhal pour les larges pantalons que les dames passent gracieusement par-dessus leurs robes, est remplacée selon l'annonce touristique par la «tenue de mineur blanche» qu'il faut enfiler pour les fameuses glissades :

Vous passerez d'un niveau de la mine à l'autre en glissant sur des toboggans. Un ancien mineur vous racontera l'histoire du sel et vous traverserez le lac souterrain sur un radeau aménagé, bercé par une musique mystique ${ }^{\text {sI }}$.

Le prospectus du site montre les troncs lisses et polis qu'on enfourche pour atteindre une vitesse de soixante kilomètres-heure. On imagine le sourire moqueur de Stendhal à la promesse de cette "musique mystique», un tropisme bien germanique!

La première fois, il y va ganz allein («tout seul») : «Le commissaire des guerres de Beyle» comme on peut lire à la date du 5 janvier. Mais, le lendemain, il y est retourné, jouant sans doute son rôle favori de cicerone auprès de ses collègues (Fromentin, Delaunay, etc.), gazouillant en son anglais qui est parfois du grec ou de l'allemand pour les éditeurs durs d'oreille, la mention : «coming to France ${ }^{52}$ ».

50. R. Baehr, d'ailleurs né Allemand (du Sud!) à Bamberg en Bavière : merci à Chr. Weiand, notre "ami dans la langue d'ya " pour cette nuance. Voir R. Baehr, art. cité, ainsi que : K. Ringger, R. Baehr, Chr. Weiand, Stendhal und Österreich, Stendhal-Hefte 3, Tübingen, I989. 5I. De l'Amour, ouvr. cité, chap. viI, p. I25.

52. Ainsi, dans un passage anglais du Journal: «j'ai beaucoup travaillé to my letters for Dutchland» (cahier 4 de Berès, 29 décembre I8IO), corrigé absurdement par toutes les éditions 
Ces documents sont reproduits dans Stendhal Club. Une telle curiosité ne nous surprend pas de sa part. On sait par une note de I8Is qu'il est descendu précédemment aux mines de plomb, zinc et argent de Clausthal-Zellerfeld dans le Harz pendant sa période brunswickoise (I807-1808) avec Héron de Villefosse (I774-1852). Ce dernier est mentionné laudativement dans le petit guide en français du musée local pour les années I807-I8I3 : «Domination française : exploitation des mines sous la direction éminente de Héron de Villefosse. " Là aussi, lacune du Journal, ce cahier perdu comme on sait par Stendhal lui-même dans ces étranges années d'apprentissage et de voyage, Lehrjahren, Wanderjahren, pour reprendre le vocabulaire goethéen du Wilhelm Meister, ici pertinent. Or cette note rétrospective (I8I5, sur 1808) évoque une petite société en visite aux mines, où il $\mathrm{y}$ avait des dames :

Le fredetto envers les femmes de M. Héron de Villefosse à Clausthal - Ce caractère est le plus opposé possible à celui de Dominique ${ }^{53}$.

La mise en scène finale du "rameau de Salzbourg», en vue d'une seconde édition de son livre favori, avec odor di femina - en présence de Madame Gherardi, qui n'y était «sûrement pas» elle, dit encore Martineau décidément rageur! Certes! Cette fois, nous l'admettons! - donc cette récriture des trois brefs paragraphes du chapitre in de De l'Amour sur la "cristallisation" nous semble s'inspirer autant du voyage à Clausthal-Zellerfeld dans le Harz au temps de Brunswick, que de la double montée-descente dans la mine de sel à Hallein. Celle-ci coïncide avec la lecture enthousiaste des Affinités électives parues en I809, "comme une circulaire à mes amis" disait Goethe et aussitôt traduites en français. Beyle se situe d'emblée parmi ces happy few. Il est amoureux de l'héroïne Odile (Ottilie en allemand) comme le montre sa lettre à Pauline du I4 février I8IO : "J'ai été tendrement ému hier soir, tu connaîtras Ottilie.» En mai, à Paris, il ira encore porter "Ottilie, à Mme Z», Alexandrine Daru. La jeune fille du roman se révèle mystérieusement sensible aux filons métalliques souterrains dont elle ignore l'existence. Ce détail

en Deutschland depuis Champion; lettres pour la Hollande comme on le sait en détail depuis les travaux d'E. Williamson rassemblés dans Stendhal et la Hollande (Institute of Romance Studies, University of London School of Advanced Studies, 1996). Dans le même cahier peu avant, Amyot (Amiot) est nommé chez les Bataves, "in Dutch» dans le texte, et non en Deuts(chland) (8 octobre I8Io). Ce qu'avait déjà compris par le contexte Chr. Thompson (voir Stendhal et la Hollande, ouvr. cité, "Introduction», p. XX).

53. Stendhal, Mélanges intimes et Marginalia, t. I, H. Martineau, Le Divan, 1936, p. 376. Chargé alors notamment des mines du Royaume de Westphalie. Stendhal écrit d'ailleurs "Clausdhal» avant de s'inventer «Stendhal». Villefosse, mentor de Beyle à Brunswick, prescrivait un "vernis de freddo sans lequel auprès des femmes on joue toujours le rôle d'Oreste». Il se souviendra de cette excellente "maxime» écrite, dit-il, "sur mes pantoufles» (CEuvres intimes, t. I, p. 602). 
parmi d'autres, et bien sûr la métaphore chimique des «affinités» initialement projetée sur le quatuor amoureux, inscrivent la sympathie de Stendhal dans la Spätaufklärung : ce concept de "Lumières tardives" nullement péjoratif, convient autant à la singularité élitiste du roman de Goethe qu'à la production populaire d'un August Lafontaine, non moins chère à Stendhal pour cette raison même. Le goût des sciences de la nature est en tension avec une exploration précise du sentiment inscrit dans son incarnation physique : en somme, "exactitude et âme ${ }^{54}$ » comme dira ironiquement Musil dans L'Homme sans qualités, Musil, grand lecteur de Goethe et de Stendhal, et lui aussi solidement adossé à une culture scientifique.

Les riches arômes des millésimes I809 et I8IO vont s'ouvrir peu à peu au fil du temps dans l'écriture de Stendhal. Nous retiendrons trois moments.

D'abord une pierre blanche marque le premier livre imprimé. La lettre XX des Vies de Haydn, Mozart et Métastase, qui porte sur les Saisons de Haydn, est datée sans raison apparente de: "Halein, le 5 juin 180955 ".

Le $5 \mathrm{a}$ a-t-il ici un sens mémoriel pour Stendhal, comme réminiscence du 5 janvier I8Io? Le mois de juin est imposé par la chronologie interne des lettres supposées. Le nom de Hallein a perdu un l, mais il est bien en évidence, le lieu et la date de la lettre sont en position ostensible d'épigraphe dans ce chapitre qui introduit aussi en note une lettre fictive sur «l'espèce de goût que Werther avait pour la musique», qui rappelle l'expérience brunswickoise des «jardins anglais» avec leurs couples d'amants

prenant du café à côté de leurs parents tandis que des troupes de musiciens bohèmes jouent avec leurs cors leurs valses et leur musique lente et si touchante ${ }^{56}$.

L'allusion au Voi che sapete de Chérubin prépare ici l'épigraphe du Rouge: Non so più cosa son, cosa facio pour la rencontre amoureuse (I, 6). L'opéra de Mozart Le Nozze di Figaro entrelace la musique "allemande» (autrichienne, c'est la première école de Vienne?) à la langue italienne. Quoi de plus heureux ici pour la pensée de Stendhal qui se cherche? Le plagiaire de Carpani n'hésite pas à enrichir son texte du "fragment d'une lettre d'Ottilie» prétendument extraite des Affinités électives: Cimarosa, pour le Matrimonio segreto et les Horaces, le Prométhée de Vigano s'invitent alors dans le commentaire de la Création de Haydn, avec une reprise du fil rouge gothéen en fil d'or ${ }^{57}$. Ces

54. Voir le titre du chapitre in6 du Premier livre de L'Homme sans qualités : «Les deux arbres de la vie et la demande d'un Secrétariat général de l'Exactitude et de l'Âme».

55. Vies de Haydn, de Mozart et de Métastase, éd. V. Del Litto, Genève, Cercle du Bibliophile, p. $2 \mathrm{I} 3$.

56. Ibid., p. 216-218.

57. "C'est une idée allemande que je vais vous présenter. Je lis dans Ottilie ou les Affinités électives de Goethe [...]»(Ibid., p. I83-185). 
deux lettres montrent à l'évidence que les Vies de Haydn, Mozart et Métastase sont moins un plagiat qu'un recueil d'exercices de style très personnels, qui prennent plus d'une fois la forme de lettres intimes. Déjà Stendhal perce sous Bombet.

Dans un second temps, relisant les cahier du Journal sans omettre, on ose dire, leurs lacunes, De l'Amour utilise la description dense, précise et poétique, d'une expérience, d'un protocole scientifique, comme métaphore de la "cristallisation" amoureuse, dans un mouvement hypothético-déductif sur un schéma a-b-a, et une forme sonate, pour en donner la définition :

Laissez travailler la tête d'un amant pendant 24 heures et voici ce que vous trouverez: Aux mines de sel de Salzbourg, on jette dans les profondeurs abandonnées de la mine un rameau d'arbre effeuillé par l'hiver; deux ou trois mois après, on le retire couvert de cristallisations brillantes : les plus petites branches, celles qui ne sont pas plus grosses que la patte d'une mésange, sont garnies d'une infinité de diamants mobiles et éblouissants; on ne peut plus reconnaître le rameau primitif. Ce que j'appelle cristallisation, c'est l'opération de l'esprit, qui tire de tout ce qui se présente la découverte que l'objet aimé a de nouvelles perfections ${ }^{58}$.

Les saisons ne concernent pour le moment, dans ce premier temps d'écriture, que le rameau : l'hiver au temps de sa catabase, le printemps ou un début d'été quand la lumière solaire enchante la remontée des cristaux formés dans les profondeurs de la terre. C'est le texte de i822.

Le troisième temps est celui du fragment de 1825 intitulé Le Rameau de Salzbourg, si précis dans ses realia, splendidement réécrit en vue d'une seconde édition qui sera posthume de De l'Amour, dans la première édition collective Lévy. Stendhal prend à rebours sa topique du Sud désiré et de l'altitude euphorisante, évincés au profit du Nord et des profondeurs de cette mine de sel gemme, qui franchit souterrainement une frontière austro-allemande artificielle autant que récente... Quant à la précision géographique, l'auteur peut, comme nous, consulter les cartes et guides de l'époque. S'il ne se souvient pas personnellement de Golling, des sources accessibles lui rafraîchiront aisément la mémoire. On lit partout que la cascade vertigineuse et pittoresque de Golling vaut le voyage. Ici, les voyageurs viennent d'un Gothard lointain, et non du Brenner comme on pourrait s'y attendre, avec une ellipse très stendhalienne des distances. Mais c'est une fiction construite sur des réalités. Le plus étonnant pour la vulgate stendhalienne, c'est que le Nord devienne désirable : car on s'en va "prendre le frais au mont Saint-Gothard", pour fuir la canicule de Bologne :

La fraîcheur charmante de ce revers des Alpes, du côté du Nord, comparée à l'air étouffé et à la poussière que nous venions de laisser dans la plaine de Lombardie

58. Stendhal, De l'Amour, ouvr. cité, chap. II, p. 8-9. 
nous donnait chaque matin un plaisir nouveau et nous engageait à pousser plus avant. Nous achetâmes des vestes de paysans à Golling ${ }^{\varsigma 9}$.

Donc c'est le plein été solaire, tempéré par l'altitude - le 3 août, la simple "promenade» est devenue tout un "voyage» euphorique, à bottes de sept lieues more stendhaliano. On s'habille comme les gens du cru, le dépaysement n'est pas un exil. Car l'espace est en miroir de part et d'autre des Alpes. Les luoghi ameni du pays salzbourgeois sont comme une Brianza du Nord qui invite elle aussi à prendre la plume, "a empir le carte $e^{60}$ ". On verra cinq ans plus tard un effet analogue autour du lac du Bourget dans la Savoie frontalière de Mina de Vanghel ${ }^{6 .}$. Ici, un jeune Allemand du sud, officier bavarois, "cristallise» pour la belle Italienne. La société est conviée à l'observation du gracieux phénomène. L'auteur du «rameau de Salzbourg», avec ses souvenirs de Virgile, du rameau d'or et du mythe de Proserpine, donne forme à un nouveau mythe très personnel. La place du divin y est prise par les puissances de l'esprit humain et de la terre qui le porte. Stendhal mesure le chemin parcouru. Il n'est plus le rédacteur un peu vert du Journal de Brunswick (I807 à I808) qu'il relit périodiquement. Ses relectures portent des appréciations progressives et nuancées, d'abord en I8I5: "content de ce cahier»; puis en juin I820, alors qu'il écrit De l'Amour et notamment les chapitres sur le mariage : «assez content : j'errais encore au hasard, faute d'éducation». Une pensée se construit, et la seconde des trois préfaces du traité insiste sur l'intellectualité du propos. L'ouvrage exige du lecteur qu'il prenne l' «habitude de penser en lisant». Ce livre étrange, cet essai inclassable, pourrait être comme chez Montaigne ou Rousseau "quelqu'un de ces ouvrages insolents qui forcent le lecteur à penser». L'esprit de Rome, Naples et Florence (1827) est déjà là, on reconnaît d'ailleurs au passage sa future épigraphe : «Nos gens de Paris qui en sont encore à concevoir qu'on puisse être Persan $[\ldots]^{62}$."

La grande nouveauté, et Stendhal désormais s'y tiendra, c'est une tension, un équilibre affichés, revendiqués, entre les expériences allemande et italienne. On perçoit cet écho entêtant de son adieu à l'Autriche dans la préface de 1826 pour son livre préféré (la première d'une série de trois, toujours pour une seconde édition, il y tient) : "J'allai avec Madame Gherardi aux mines de

59. Le Rameau de Salzbourg, ouvr. cité, "Dossier», p. I9I.

6o. L'épigraphe de la Chartreuse cite l'Arioste : "Jadis des lieux charmants me furent une douce invitation à écrire.»

6I. Voir Stendhal, Le Rose et le vert, Mina de Vanghel, Tamira Wanghen, éd. J.-J. Labia, Flammarion, coll. "GF", I998, «Introduction», p. 43.

62. De l'Amour, ouvr. cité, chap. XuIx, p. 156. L'épigraphe de Rome, Naples et Florence (I827) resserre le texte de Montesquieu (Lettres persanes) : "Ah! Monsieur, comment peut-on être Persan?» Comparer avec la pointe de la lettre XXX dans le texte original : "Ah! Ah! Monsieur est Persan? C'est une chose bien extraordinaire! Comment peut-on être Persan?» 
Hallein" - dit l'auteur, son encre est à peine sèche car il vient d'écrire cette seconde version du «rameau de Salzbourg" - l'auteur "qui a passé quinze ans en Allemagne et en Italie». Le Stendhal des Souvenirs d'égotisme bâtira en I832 une symétrie analogue dans le domaine de l'expérience musicale autre apprentissage, autre "éducation", si l'on se souvient du mot précité de I820 : «Enfin, j'ai adoré la musique et avec le plus grand bonheur pour moi de I806 à I8Io en Allemagne. De I8I4 à I82I en Italie ${ }^{63}$.» On voit le prix qu'il veut accorder à ces quelques jours ultimes de janvier I8Io encore passés en Autriche, "coming to France» comme il l'écrit inimitablement sur place et dans le livre, au point de les étirer jusqu’à une année!

En I8Io, Stendhal encore épris d'Alexandrine Daru, «Mme $Z$ » va bientôt lui offrir à Paris, comme on l'a vu, l'un des trésors rapportés de son année autrichienne, les Affinités électives de Goethe. Il joue à prolonger son séjour dans une Allemagne littéraire. En témoigne sa découverte émerveillée du Wilhelm Meister ${ }^{64}$. Feder en portera la trace ${ }^{65}$. Dans les bras parisiens de «Frau Mozart", Angeline Bereyter, son "actrice chantante» il retrouve un parfum de Vienne. Frau Babet Rothe, du Theater an der Wien le hante encore ${ }^{66}$. Pour Stendhal, l'Autriche prolonge la signature allemande, comme un gracieux paraphe qui flirte avec le Sud italien.

À l'instant du retour, le contraste est frappant avec cet autre voyageur, le chevalier Cadet de Gassicourt, Premier pharmacien de l'Empereur, bientôt membre de plusieurs académies, bourgeoisement content de retrouver ses amis, ses pantoufles, sa famille et ses enfants - car de sa femme il ne dit mot :

En rentrant en France [...] je reconnais tout le mérite de ce vers :

Plus je vis d'étrangers, plus j'aimais ma patrie

Tout me parait plus aimable, plus vivant, plus humain qu'en Allemagne.

J'éprouve l'étonnement agréable d'un homme qui s'éveille fatigué d'un long rêve ${ }^{67}$.

63. Stendhal, Souvenirs d'égotisme, ouvr. cité, p. 494.

64. Cahier 3 de Berès, mai-août 1810 , fos 91 et 92 , en ligne, Bibliothèque municipale de Grenoble : "J'ai joui ensuite avec Göthe, near to my soul. J'ai fini les années d'apprentissage de W. ${ }^{\mathrm{em}}$ Meister, ces idées m'avaient presque rendu fou, et c'est dans cette disposition que j'ai commencé à écrire.» Le mot «presque» est absent des éditions Martineau et Del Litto. Plus d'un détail reste à amender dans la lettre même des "journaux et papiers", selon l'intitulé choisi par l'équipe de Grenoble.

65. Le héros, fils d'un marchand bavarois expatrié à Marseille, préfère épouser une jeune actrice et partir suivre sa vocation d'artiste, sur les pas de Wilhelm Meister à sa façon. Il porte un nom d'un philosophe (adversaire de Kant, cité notamment par Schopenhauer) que Stendhal a pu ramener dans son bagage brunswickois, car il enseignait à Göttingen : Johann Georg Feder (1740-I82I).

66. Voir R. N. Coe, Lisbeth folle par amour, ou les soirées d'un commmissaire des guerres, Stendhal Club, 39-40, 1967-1968.

67. Ch.-L. Cadet de Gassicourt, Voyage en Autriche..., ouvr. cité, p. 232. 
Recherches \& Travaux - $\mathrm{n}^{\circ} 79$

Il oublie les nuances, il croit qu'il est allé en Allemagne. Ce n'est pas entièrement faux, on l'a vu. Mais il est à l'exact opposé de l'étonnement stendhalien du retour :

Le plus grand avantage du voyage est peut-être qu'au

retour, on sétonne

de ce qu'on voit dans son pays ${ }^{68}$

68. C’est Stendhal qui souligne le mot sur un exemplaire interfolié. Voir J.-J. Labia, Rome, Naples et Florence (I826) : les notes et variantes de l'exemplaire Filippi, L'Année stendhalienne, $\mathrm{n}^{\circ}$ 2, Honoré Champion, 2003, p. 330. Quoi de plus persan? 